\title{
Engineered synthetic scaffolds for organizing proteins within the bacterial cytoplasm
}

\author{
Matthew J Lee', Judith Mantell ${ }^{2,3}$, Lorna Hodgson ${ }^{2}$, Dominic Alibhai ${ }^{3}$, Jordan M Fletcher ${ }^{4}$, \\ Ian R Brown', Stefanie Frank ${ }^{5}$, Wei-Feng Xue ${ }^{1}$, , Paul Verkade ${ }^{2,3,6}$, Derek N Woolfson ${ }^{2,4,6 \star}$ \\ \& Martin J Warren ${ }^{1}$ [ *
}

\begin{abstract}
We have developed a system for producing a supramolecular scaffold that permeates the entire Escherichia coli cytoplasm. This cytoscaffold is constructed from a three-component system comprising a bacterial microcompartment shell protein and two complementary de novo coiled-coil peptides. We show that other proteins can be targeted to this intracellular filamentous arrangement. Specifically, the enzymes pyruvate decarboxylase and alcohol dehydrogenase have been directed to the filaments, leading to enhanced ethanol production in these engineered bacterial cells compared to those that do not produce the scaffold. This is consistent with improved metabolic efficiency through enzyme colocation. Finally, the shell-protein scaffold can be directed to the inner membrane of the cell, demonstrating how synthetic cellular organization can be coupled with spatial optimization through in-cell protein design. The cytoscaffold has potential in the development of next-generation cell factories, wherein it could be used to organize enzyme pathways and metabolite transporters to enhance metabolic flux.
\end{abstract}

(R

$\mathrm{n}$ industrial biotechnology and synthetic biology there is a growing need to generate internal supramolecular scaffolds in bacteria en route to delivering so-called cell factories ${ }^{1}$. To this end, researchers have investigated protein-based linkers ${ }^{2}$, lipids ${ }^{3}$ and nucleic acids ${ }^{4,5}$ as modulators to attain high-level biomolecular organization. However, none of these approaches have delivered a uniform matrix throughout the bacterial cytoplasm. The advantage of such scaffolding systems is that they can be used to direct and align biosynthetic pathway enzymes to orchestrate greater production of commodity and specialty chemicals, especially in pathways that proceed through unstable or toxic intermediates ${ }^{6,7}$. This is because the close proximity of enzymes on a scaffold allows greater channeling of intermediates through improved flux, stabilization of intermediates and protection from other reactions $s^{8,9}$.

A number of natural scaffolds are found in bacterial cells. For instance, bacterial microcompartments (BMCs) are organelles with an outer semipermeable scaffold in the form of a protein shell, which encases a specific metabolic pathway ${ }^{10-12}$. BMCs have a diameter of approximately $150 \mathrm{~nm}$ and possess high concentrations of internalized enzymes. This is most apparent in carboxysomes, which are anabolic BMCs wherein the high concentrations of carbonic anhydrase and ribulose-1,2-bisphosphate carboxylase/oxygenase (RuBisCO) ensure enhanced carbon fixation ${ }^{13,14}$. In catabolic BMCs, such as the metabolosome associated with propanediol utilization (the so-called Pdu system), internalized enzymes necessitate that propionaldehyde be rapidly transformed into either an alcohol or a CoA thioester, thereby protecting the cell from the potentially toxic aldehyde intermediate ${ }^{15-17}$. In all cases, the enzymes are targeted to the interior of the BMCs by small encapsulation peptides, which interact with a component of the outer-shell scaffold ${ }^{18-21}$. Modeling studies indicate that BMCs enhance flux through intermediate sequestration ${ }^{22}$. Recently, a detailed structure of a recombinant BMC shell has been reported showing the precise orientation of the different shell proteins that tile together to form the outer casing, providing molecular detail on how the shell proteins scaffold together to act as a semipermeable membrane ${ }^{23}$.

Apart from BMCs, the other major scaffold within prokaryotic cells is the cytoskeleton ${ }^{24}$, which is generally distributed around the inner membrane. This filamentous structure, which includes proteins such as FtsZ, MreB, ParM and MinD ${ }^{25,26}$, has roles in cell division, cell morphology and structural polarity. However, the essential nature of these proteins precludes them from being developed as major cellular matrices. For these reasons, we sought to construct a simple and modular bacterial cytoskeleton, which we call a cytoscaffold, using components that we understand and can manipulate predictably.

Previously, we have shown that a single shell protein from the Citrobacter freundii Pdu BMC, with a minor modification to its $\mathrm{C}$ terminus to improve solubility ( $\mathrm{PduA}^{*}$ ), forms filaments in $E$. coli ${ }^{27}$. PduA itself hexamerizes to form a tile that assembles to make the facets of the BMC casing ${ }^{28,29}$. However, when overproduced recombinantly in E. coli, $\mathrm{PduA}^{\star}$ forms hollow filaments approximately $20 \mathrm{~nm}$ in diameter that span the length of the cell ${ }^{30}$. Moreover, these structures often interfere with septation during cell division. Nonetheless, we reasoned that PduA*-based filamentous structures may present tractable scaffolds for tethering other proteins.

Here, we describe a three-component system comprising PduA* and two complementary de novo-designed coiled-coil peptides ${ }^{31}$, which form an interactive intracellular filamentous arrangement that gives the appearance of a matrix permeating the entire E. coli cytoplasm (Supplementary Results, Supplementary Fig. 1). We show that other proteins can be specifically targeted to these cytoscaffolds. Building on this, we demonstrate that tethering metabolic enzymes for ethanol production to the $\mathrm{PduA}^{\star}$ scaffold increases their effective local and relative concentrations and results in improved ethanol production. Finally, we show that the scaffold can be directed

IIndustrial Biotechnology Centre, School of Biosciences, University of Kent, Canterbury, UK. ${ }^{2}$ School of Biochemistry, University of Bristol, Medical Sciences Building, University Walk, Bristol, UK. ${ }^{3}$ Wolfson Bioimaging Facility, Medical Sciences Building, University Walk, Bristol, UK. ${ }^{4}$ School of Chemistry, University of Bristol, Cantock's Close, Bristol, UK. 5 Department of Biochemical Engineering, University College London, Bernard Katz Building, Gordon Street, London, UK. ${ }^{6}$ BrisSynBio, Life Sciences Building, Tyndall Avenue, Bristol, UK. *e-mail: M.J.Warren@kent.ac.uk or D.N.Woolfson@bristol.ac.uk 
to the inner membrane of the cell, further illustrating its modularity, flexibility, and utility, and demonstrating how synthetic cellular organization can be coupled with spatial optimization.

\section{RESULTS}

\section{Construction of a filamentous scaffold}

Initially, we tested whether different proteins could be recruited to $\mathrm{PduA}^{*}$ filaments in vivo in an analogous way to how encapsulation peptides are thought to work in natural BMCs (see above). Attempts to use the natural encapsulation peptides themselves were not very successful, mainly owing to aggregation within the cell ${ }^{32}$. Therefore, we turned to a better-characterized de novo-designed heterodimeric coiled-coil system, CC-Di-AB ${ }^{31}$, which has been used successfully in the construction of self-assembling peptide cages $^{33}$. The heterodimer comprises two peptides (acidic (A) and basic (B)) that do not selfassociate, but interact specifically and tightly when mixed. The concept was to fuse either CC-Di-A or CC-Di-B to PduA* and then test whether a reporter protein with the cognate peptide can be targeted to the filaments. Plasmids encoding fusion proteins of the following type were made: CC-Di-A/B-Gly/Ser linker-HexaHisTag-PduA*, referred to as CC-Di-A-PduA* and CC-Di-B-PduA*. A control plasmid harboring the fusion without the CC-Di-A/B module, i.e., containing only the Gly/Ser linker-HexaHisTag $\left(\mathrm{C}-\mathrm{PduA}^{*}\right)$, was also made. Plasmids were transformed individually into $E$. coli cells, and the resulting strains were grown, induced and analyzed by transmission electron microscopy (TEM) after fixation, embedding, thin sectioning and staining.

Strains expressing $\mathrm{PduA}^{*}$ alone generated parallel filaments spanning the length of the cell (Fig. 1a) and appear to interfere with septation (Supplementary Fig. 2). Unexpectedly, the strains producing the control C-PduA ${ }^{*}$ and the CC-Di-A-PduA ${ }^{*}$ did not form any filamentous structures (Fig. $1 \mathbf{b}$ and Supplementary Fig. 3). C-PduA* expression led to deposits of material at the poles of the cell, suggesting that the Gly/Ser linker and/or the hexahistidine tag alone affects solubility of the fusion protein. This was not seen with CC-Di-A-PduA ${ }^{*}$, but it is not clear why filaments do not form with this construct. In both cases, western blot analysis revealed only low levels of CC-Di-A-PduA ${ }^{*}$ and $\mathrm{C}-\mathrm{PduA}^{*}$ relative to untagged $\mathrm{PduA}^{*}$, suggesting potential cytotoxicity of these proteins (Supplementary Fig. 4). In contrast, large amounts of CC-Di-B-PduA ${ }^{*}$ were detected (Supplementary Fig. 4), and this led to numerous filaments throughout the cytoplasm (Fig. 1c and Supplementary Fig. 5). These filaments had a diameter of $23.6 \pm 2.78 \mathrm{~nm}(n=100)$, similar to those of untagged PduA $^{*}$ filaments ${ }^{30}$, but the former were considerably shorter (Fig. 1d,e). Consequently, CC-Di-B-PduA* filaments were not aligned within cells, and they did not appear to disrupt cells. It is not clear why the CC-Di-B-PduA* filaments are shortened, but it is possible that the highly charged CC-Di-B peptide may limit filament growth in some way.

\section{Characterization of the cytoscaffold}

To probe the spatial localization and organization of the shorter CC-Di-B-PduA filaments, thicker thin sections $(250 \mathrm{~nm})$ were cut and prepared for TEM tomography. Analysis of the resulting tomogram confirmed the presence of the shorter filaments throughout the cytoplasm, except in a central region that is largely occupied by genomic DNA (Fig. 1f). Using methods developed to track microtubule assemblies in cells ${ }^{34}$, we rendered these structures and visualized them in three dimensions (Fig. If and Supplementary Videos 1 and 2). From this, it was clear that the filaments were not aligned, but arrayed with multiple orientations, resulting in the appearance of an internal matrix. Analysis of these filaments revealed an average length of $161.2 \pm 102.4 \mathrm{~nm}(n=739)$; though because of the limitation of a $250 \mathrm{~nm}$ thin section, the true length is likely longer than this (Fig. 1e).
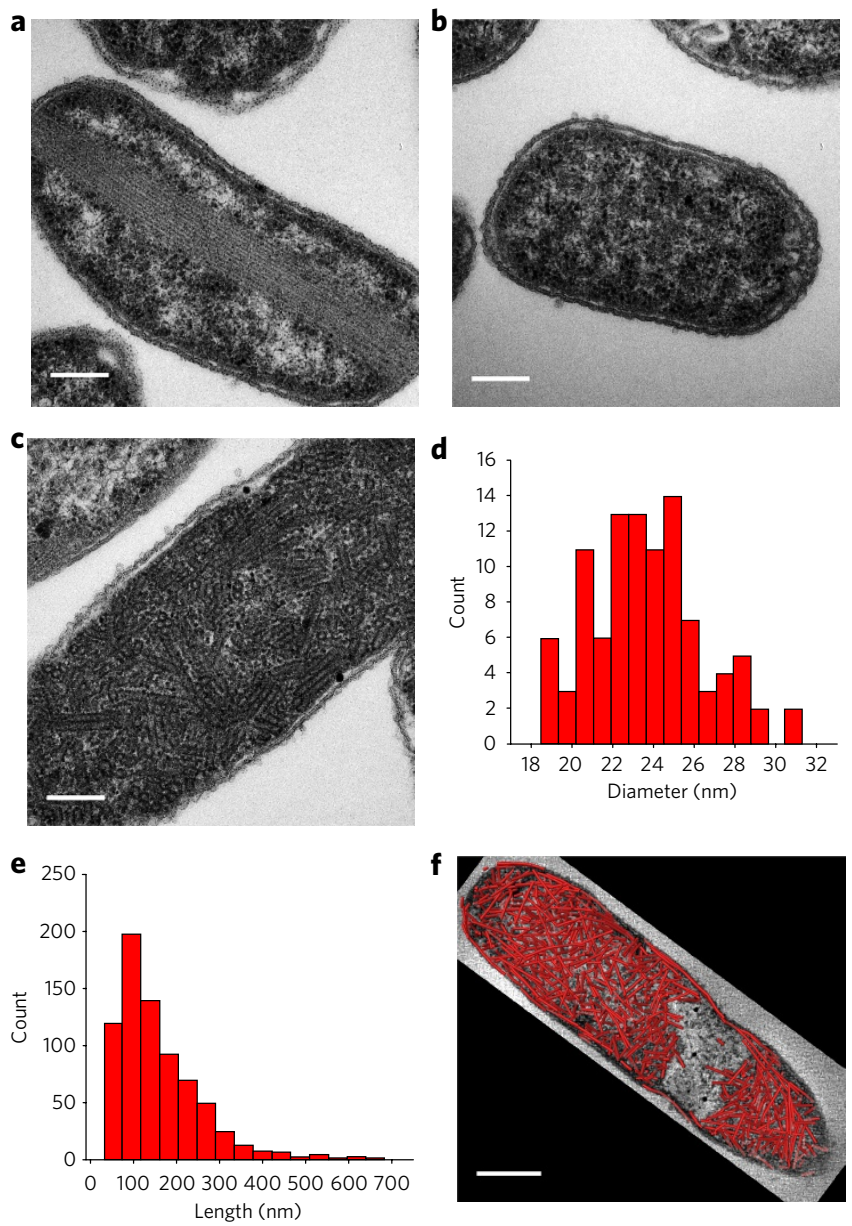

Figure 1 | Transmission electron micrographs and analysis of PduA $A^{\star}$ based constructs and filaments in $\boldsymbol{E}$. coli. (a) Untagged PduA* filaments. $(\mathbf{b}, \mathbf{c})$ CC-Di-A-PduA* (b) and CC-Di-B-PduA* (c) filaments. Scale bars in $\mathbf{a}-\mathbf{c}, 200 \mathrm{~nm}$. (d) Histogram showing the diameter of CC-Di-B-PduA* filaments ( $n=100$ measurements). (e) Histogram showing lengths of CC-Di-B-PduA* filaments based on a $250 \mathrm{~nm}$ tomogram rendering shown in $\mathbf{f}\left(n=739\right.$ filaments). (f) $3 \mathrm{D}$ rendering of CC-Di-B-PduA $A^{*}$ filaments in E. coli based on a $250 \mathrm{~nm}$ tomogram. Scale bar in $\mathbf{f}, 500 \mathrm{~nm}$. See also Supplementary Video 1.

To test the robustness of the CC-Di-B-PduA* filaments, and to interrogate their structure in more detail, we purified the filaments from cells using protocols developed for BMC isolation ${ }^{21}$. Cells were lysed, and the filaments were purified by combining centrifugation and differential salt precipitation (Supplementary Fig. 6). Purified filaments were analyzed by TEM and atomic force microscopy (AFM). Both confirmed intact filaments, and these approaches provided the opportunity to gain greater insight into the molecular organization of these structures (Supplementary Fig. 7). These ex vivo filaments tended to cluster together on the TEM grids and AFM substrates. This clustering was also seen, though to a lesser extent, in some thin sections of whole cells visualized by TEM.

\section{Targeting to and functionalization of the cytoscaffold}

Next, we tested whether the CC-Di-B peptides of the CC-Di-B-PduA* filaments were accessible for targeting by other proteins labeled with CC-Di-A using the fluorescent protein Citrine. To do this, we made CC-Di-A-Citrine and C-Citrine constructs similar in design to the fusion proteins described above. By cloning these constructs in compatible plasmids, we could either transform these alone or co-transform them with the plasmid producing CC-Di-B-PduA* 



Figure $\mathbf{2}$ | Localization of fluorescent proteins to a bacterial cytoscaffold. (a) Co-expression of CC-Di-B-PduA* with CC-Di-A-Citrine, indicated by Citrine fluorescence. (b-e) Correlative light electron microscopy of a strain expressing CC-Di-B-PduA* and CC-Di-A-GFP, encompassing TEM (b), GFP fluorescence (c), overlay (d), and detail of the overlaid image (e). (f-h) Fluorescence of cells expressing CC-Di-B-PduA*, CC-Di-A-Citrine, and CC-Di-A-mCherry, including Citrine signal (f), mCherry signal ( $\mathbf{g})$, and overlay of the two (h). Scale bars in a,f-h, $5 \mu \mathrm{m}$; scale bars in $\mathbf{b}-\mathbf{e}, 500 \mathrm{~nm}$.

On their own, CC-Di-A-Citrine and C-Citrine each produced uniform fluorescence throughout the cells, consistent with soluble, cytoplasmic proteins (Supplementary Fig. 8). Similarly, when coexpressed with CC-Di-B-PduA ${ }^{*}$, the C-Citrine control produced fluorescence that was distributed throughout the cell. In contrast, co-expression of CC-Di-A-Citrine and CC-Di-B-PduA* produced more punctate fluorescence, as well as reduced fluorescence around the genomic DNA (Fig. 2a). This is consistent with CC-Di-A-Citrine being localized to the filamentous scaffold. Correlative light electron microscopy (CLEM) $)^{35,36}$ of high-pressure frozen cells co-expressing



Figure 3 | Ethanol production in vivo. Graph showing ethanol content of the growth medium over time normalized to an $\mathrm{OD}_{600}=1$. Open circles, E. coli strain transformed with empty plasmids ( $\mathrm{pET14b}$ and pLysS); closed circles, strain producing CC-Di-B-PduA* only; open triangles, strain producing CC-Di-A-Pdc and CC-Di-A-Adh; closed triangles, strain producing CC-Di-A-Pdc, CC-Di-A-Adh and CC-Di-B-PduA*. Data points represent an average of three independent experiments; error bars represent s.d.

CC-Di-A-GFP and CC-Di-B-PduA ${ }^{*}$ confirmed the localization of fluorescence to the intracellular filamentous network (Fig. 2b-e). Control strains expressing the CC-Di-B-PduA* filaments with untagged GFP showed only a cytoplasmic signal (Supplementary Fig. 9). Expression of CC-Di-B-Citrine with or without CC-Di-B$\mathrm{PduA}^{*}$ resulted in punctate fluorescence, suggesting self-association of the CC-Di-B peptide (Supplementary Fig. 8).

To demonstrate that multiple cargo proteins can be directed to the cytoscaffold, we co-produced CC-Di-A-Citrine and a CC-DiA-mCherry fusion in cells with CC-Di-B-PduA* filaments. This gave patterns similar to those observed with CC-Di-A-Citrine plus CC-Di-B-PduA*, and the mCherry signals colocalized with the Citrine signals (Fig. 2f-h).

Although the results clearly demonstrate that fluorescent proteins can be localized to the $\mathrm{PduA}^{*}$ filaments through the use of the coiled-coil interaction, we also wanted to investigate whether enzymes could be pegged onto the CC-Di-B-PduA* filaments in a similar fashion. To explore this, we tagged both pyruvate decarboxylase (Pdc) and alcohol dehydrogenase (Adh) with the CC-Di-A peptide and co-expressed these with and without the CC-Di-B-PduA* filaments. Intriguingly, strains expressing Pdc and Adh grew to a substantially higher optical density at $600 \mathrm{~nm}$ $\left(\mathrm{OD}_{600}\right)$ in comparison to both control strains (Supplementary Fig. 10a). GC-MS analysis of the growth medium revealed that the introduction of the CC-Di-B-PduA ${ }^{*}$ filamentous network increased ethanol production by $221 \%$ per OD unit $(t=120 \mathrm{~h})$ in comparison to a strain expressing Pdc and Adh, but not the cytoscaffold (Fig. 3 and Supplementary Fig. 10b). Western blot analysis showed that this increase in ethanol production was not due to increased protein expression: indeed, the levels of Pdc and Adh were actually reduced by $48 \pm 13.3 \%$ and $26 \pm 5.5 \%$, respectively, in the strain expressing CC-Di-A-tagged enzymes in the presence of the CC-Di-B-PduA* filaments (Supplementary Fig. 11). The presence of filaments in these strains was confirmed by TEM analysis (Supplementary Fig. 12). These experiments provide strong evidence that the localization of enzymes onto the $\mathrm{PduA}^{*}$ scaffold substantially enhances an engineered metabolic pathway. 
a
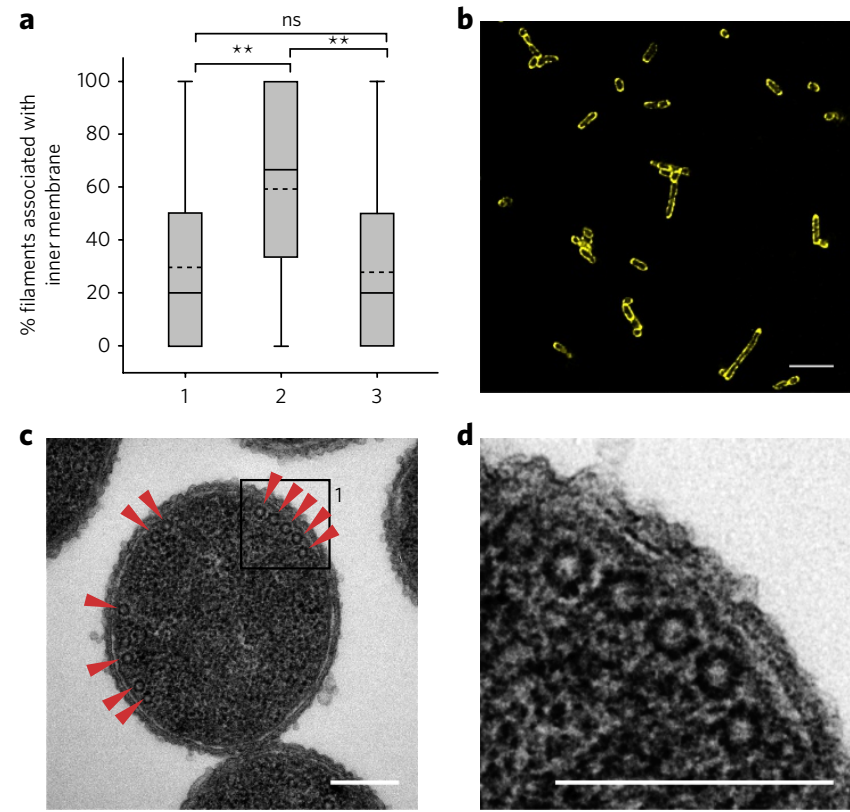

d

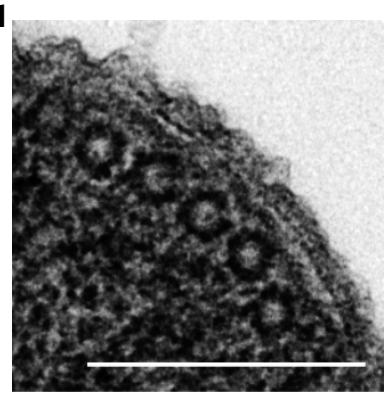

Figure 4 | Targeting the bacterial cytoscaffold to the inner membrane of $\boldsymbol{E}$. coli. (a) Box-and-whisker plots showing the number of filaments associated with the inner membrane for three strains expressing variants of the CC-Di-A/B-PduA* system. Plot $1=$ CC-Di-B-PduA*; plot $2=$ CC-DiB-PduA* + CC-Di-A-Citrine-MinD; plot $3=$ CC-Di-B-PduA* + CC-Di-CCitrine-MinD. Boxes show first and third quartiles, solid line shows median, dotted lines give the mean and whiskers the minimum and maximum; 250 cells were analyzed for each of the three strains. ${ }^{\star \star} P=0.01$; ns, not significant. (b) Confocal image of strain expressing CC-Di-A-Citrine-MinD. Scale bar, $5 \mu \mathrm{m}$. (c) TEM micrograph of strains producing CC-Di-B-PduA* plus CC-Di-A-Citrine-MinD. Arrows indicate transverse filaments. (d) Zoom in of area 1 in c (inset black box). Scale bars in c and d, $200 \mathrm{~nm}$.

As a final demonstration of the modularity, versatility and potential utility of the new cytoscaffold, we tested whether it could be directed to the cytoplasmic side of the inner membrane of E. coli (Fig. 4). For this, we added the C-terminal membrane-localizing region of MinD from Bacillus subtilis to the CC-Di-A-Citrine fusion to render CC-Di-A-Citrine-MinD ${ }^{37}$. When this construct was expressed in cells and imaged by confocal fluorescence microscopy, halos around the cytoplasm were evident, indicating localization of Citrine to the cell membrane (Fig. 4b). This was also the case for the C-Citrine-MinD control (Supplementary Fig. 13). When each of these were co-expressed with CC-Di-B-PduA* filaments, we observed differences in location between control and membranetargeting constructs (Fig. 4a and Supplementary Fig. 14).

First, in cells expressing CC-Di-B-PduA* alone, an average of $30 \%$ of the filaments were associated with the membrane. For CC-Di-B-PduA ${ }^{*}$ plus the C-Citrine-MinD control, this localization was very similar $(31 \%)$. In contrast, for the CC-Di-B-PduA* plus CC-Di-A-Citrine-MinD combination, $60 \%$ of the filaments were localized to the inner membrane, and this difference was statistically significant $(P<0.01)$. One-way analysis of variance (ANOVA) showed no significant difference $(P<0.01)$ in the total number of filaments between the three strains. Collectively, these analyses demonstrated that the cellular spatial location of the CC-Di-B-PduA* filaments can be controlled by interactions with the cognate de novo-designed coiled-coil peptide.

\section{DISCUSSION}

Previously, we and others have shown that individual shell proteins, which form the hexameric tiles of the BMC casing, generate long,

filamentous macromolecular structures when overproduced in host bacterial cells $s^{30,38}$. These structures are particularly apparent with $\mathrm{PduA}^{*}$ from the Pdu BMC. The filaments formed by $\mathrm{PduA}^{*}$ are approximately $20 \mathrm{~nm}$ in diameter; they can be several microns long and have a tendency to stack together and align along the length of the cell, to the extent that they interfere with cell septation. We hypothesized that the $\mathrm{PduA}^{*}$ filaments can be formed from the selfassociation of the hexameric tiles into a protein sheet that then rolls into a nanotubule filament. We wondered whether it would be possible to target specific proteins to these filaments to generate higherorder supramolecular organization in the cell by design.

To achieve this, we have employed a heterodimeric coiled-coil system, CC-Di-A and CC-Di- $\mathrm{B}^{31}$, previously characterized and used, for example, in the de novo construction of peptide cages ${ }^{33}$. We find that whereas fusion of the CC-Di-A sequence onto PduA* resulted in low protein production and loss of filament formation, attachment of CC-Di-B to PduA* leads to the formation of much shorter filaments that are dispersed throughout the cytoplasm. The reason for the shorter filaments is not clear, but could be because of a slight frustration of hexamer packing when the positively charged CC-Di-B peptide is appended or from faster nucleation of $\mathrm{CC}-\mathrm{Di}-\mathrm{B}-\mathrm{Pdu} \mathrm{A}^{\star}$ fusions, resulting in a greater number of shorter filaments. Importantly, given the quantity of filaments that are produced throughout the cell, their formation and presence does not appear to alter cell viability or growth.

By adding the complementary CC-Di-A peptide onto fluorescent proteins, we show through imaging techniques that these tagged proteins can be recruited to the CC-Di-B-PduA* filaments, demonstrating that the filaments can act as a molecular scaffold. A key biotechnological use of scaffolds within a cell would be localization of biosynthetic enzymes in close proximity to one another to facilitate metabolic channeling. This is part of the theory behind multienzyme complexes, although in these cases, direct transfer or channeling of metabolites from one enzyme to the next also takes place. Using simple systems it has been shown that compartmentalization of pyruvate decarboxylase and alcohol dehydrogenase within a recombinant BMC improves production of ethanol from pyruvate $^{21}$. Similarly, the direct fusion of these two enzymes also results in improved flux ${ }^{39}$, indicating in both cases that having the second enzyme in close proximity to the first ensures that the unstable acetaldehyde intermediate is more efficiently converted into the alcohol. Therefore, herein we have targeted pyruvate decarboxylase and alcohol dehydrogenase to the CC-Di-B-PduA* filaments using the coiled-coil modules, producing significantly more ethanol in comparison to when the enzymes are expressed without the scaffold. This provides very strong evidence that the cytoscaffold can be used to cluster metabolic enzymes to accelerate the channeling of intermediates from one enzyme to the next.

The CC-Di-B peptide can also be used to control the localization of the PduA* filaments within the cell. This has been achieved by targeting the CC-Di-A-Citrine protein to the inner membrane by fusing on the membrane-targeting region of $\mathrm{MinD}$ to the $\mathrm{C}$ terminus of the construct. Co-expression of this CC-Di-A-Citrine-MinD protein with $\mathrm{CC}-\mathrm{Di}-\mathrm{B}-\mathrm{PduA}^{*}$ directed filaments to the innermembrane. Such localization strategies can be used to ensure that pathway-enriched filaments have ready access to metabolites that are taken up via transporters or, conversely, to ensure that products are generated near the membrane for export out of the cell.

Furthermore, the fact that the CC-Di-B-PduA* filaments are able to interact easily with either cytosolic proteins or membranetargeted proteins containing the CC-Di-A peptide suggests that the $\mathrm{N}$-terminal region of PduA must be solvent exposed. The two sides of the hexameric PduA tiles are distinguished by their concave and convex appearance. The $\mathrm{N}$ terminus of PduA, to which CC-Di-B is fused, is located on the concave side of the protein. The fact that this CC-Di-B peptide is available to interact with a CC-Di-A-tagged 
protein strongly implies that the filament formed from PduA is generated with the concave side exposed to the solvent. This agrees with the recent structure of a recombinant BMC, in which all the shell proteins were found to be oriented with the concave side facing out of the structure ${ }^{23}$.

Overall, this work demonstrates a concept for performing and evaluating rational protein design in the cell; specifically, making hybrid scaffolds comprising de novo-designed peptides and natural proteins that can be engineered on the micron scale within the $E$. coli cytoplasm. Visualization of filaments with and without appended ancillary proteins, and of those broadly distributed filaments or those localized to the inner membrane, demonstrates the potential of the system as a universal scaffold for the attachment, dispersion or localization of targeted cargo throughout the cell. We believe that these features and properties of the cytoscaffold, coupled with its ease of decoration and remodeling within cells, will enable applications in biotechnology and synthetic biology. More generally, this ability to design and engineer proteins in the cell could usher in a new era of rational protein design and engineering in vivo.

Received 31 May 2017; accepted 1 November 2017; published online 11 December 2017

\section{METHODS}

Methods, including statements of data availability and any associated accession codes and references, are available in the online version of the paper.

\section{References}

1. Polka, J.K., Hays, S.G. \& Silver, P.A. Building spatial synthetic biology with compartments, scaffolds, and communities. Cold Spring Harb. Perspect. Biol. 8, a024018 (2016).

2. Zhang, Y. et al. Using unnatural protein fusions to engineer resveratrol biosynthesis in yeast and Mammalian cells. J. Am. Chem. Soc. 128, 13030-13031 (2006).

3. Grinkova, Y.V., Denisov, I.G. \& Sligar, S.G. Engineering extended membrane scaffold proteins for self-assembly of soluble nanoscale lipid bilayers. Protein Eng. Des. Sel. 23, 843-848 (2010).

4. Delebecque, C.J., Silver, P.A. \& Lindner, A.B. Designing and using RNA scaffolds to assemble proteins in vivo. Nat. Protoc. 7, 1797-1807 (2012).

5. Zalatan, J.G. et al. Engineering complex synthetic transcriptional programs with CRISPR RNA scaffolds. Cell 160, 339-350 (2015).

6. Agapakis, C.M., Boyle, P.M. \& Silver, P.A. Natural strategies for the spatial optimization of metabolism in synthetic biology. Nat. Chem. Biol. 8, 527-535 (2012).

7. Dueber, J.E. et al. Synthetic protein scaffolds provide modular control over metabolic flux. Nat. Biotechnol. 27, 753-759 (2009).

8. Poshyvailo, L., von Lieres, E. \& Kondrat, S. Does metabolite channeling accelerate enzyme-catalyzed cascade reactions? PLoS One 12, e0172673 (2017).

9. Wheeldon, I. et al. Substrate channelling as an approach to cascade reactions. Nat. Chem. 8, 299-309 (2016).

10. Chowdhury, C., Sinha, S., Chun, S., Yeates, T.O. \& Bobik, T.A. Diverse bacterial microcompartment organelles. Microbiol. Mol. Biol. Rev. 78, 438-468 (2014).

11. Frank, S., Lawrence, A.D., Prentice, M.B. \& Warren, M.J. Bacterial microcompartments moving into a synthetic biological world. J. Biotechnol. 163, 273-279 (2013).

12. Kerfeld, C.A. \& Erbilgin, O. Bacterial microcompartments and the modular construction of microbial metabolism. Trends Microbiol. 23, 22-34 (2015).

13. Cameron, J.C., Wilson, S.C., Bernstein, S.L. \& Kerfeld, C.A. Biogenesis of a bacterial organelle: the carboxysome assembly pathway. Cell 155, 1131-1140 (2013).

14. Kerfeld, C.A., Heinhorst, S. \& Cannon, G.C. Bacterial microcompartments. Аnnu. Rev. Microbiol. 64, 391-408 (2010).

15. Bobik, T.A., Havemann, G.D., Busch, R.J., Williams, D.S. \& Aldrich, H.C. The propanediol utilization (pdu) operon of Salmonella enterica serovar Typhimurium LT2 includes genes necessary for formation of polyhedral organelles involved in coenzyme $\mathrm{B}_{12}$-dependent 1,2-propanediol degradation. J. Bacteriol. 181, 5967-5975 (1999).
16. Havemann, G.D. \& Bobik, T.A. Protein content of polyhedral organelles involved in coenzyme B12-dependent degradation of 1,2-propanediol in Salmonella enterica serovar Typhimurium LT2. J. Bacteriol. 185, 5086-5095 (2003).

17. Sampson, E.M. \& Bobik, T.A. Microcompartments for B12-dependent 1,2-propanediol degradation provide protection from DNA and cellular damage by a reactive metabolic intermediate. J. Bacteriol. 190, 2966-2971 (2008).

18. Fan, C. \& Bobik, T.A. The N-terminal region of the medium subunit (PduD) packages adenosylcobalamin-dependent diol dehydratase (PduCDE) into the Pdu microcompartment. J. Bacteriol. 193, 5623-5628 (2011).

19. Fan, C. et al. Short N-terminal sequences package proteins into bacterial microcompartments. Proc. Natl. Acad. Sci. USA 107, 7509-7514 (2010).

20. Fan, C., Cheng, S., Sinha, S. \& Bobik, T.A. Interactions between the termini of lumen enzymes and shell proteins mediate enzyme encapsulation into bacterial microcompartments. Proc. Natl. Acad. Sci. USA 109, 14995-15000 (2012).

21. Lawrence, A.D. et al. Solution structure of a bacterial microcompartment targeting peptide and its application in the construction of an ethanol bioreactor. ACS Synth. Biol. 3, 454-465 (2014).

22. Jakobson, C.M., Tullman-Ercek, D., Slininger, M.F. \& Mangan, N.M. A systems-level model reveals that 1,2-Propanediol utilization microcompartments enhance pathway flux through intermediate sequestration. PLOS Comput. Biol. 13, e1005525 (2017).

23. Sutter, M., Greber, B., Aussignargues, C. \& Kerfeld, C.A. Assembly principles and structure of a 6.5-MDa bacterial microcompartment shell. Science 356, 1293-1297 (2017).

24. Cho, H. The role of cytoskeletal elements in shaping bacterial cells. J. Microbiol. Biotechnol. 25, 307-316 (2015).

25. Cabeen, M.T. \& Jacobs-Wagner, C. Bacterial cell shape. Nat. Rev. Microbiol. 3 , 601-610 (2005).

26. Cabeen, M.T. \& Jacobs-Wagner, C. The bacterial cytoskeleton. Annu. Rev. Genet. 44, 365-392 (2010).

27. Parsons, J.B. et al. Synthesis of empty bacterial microcompartments, directed organelle protein incorporation, and evidence of filament-associated organelle movement. Mol. Cell 38, 305-315 (2010).

28. Chowdhury, C. et al. Selective molecular transport through the protein shell of a bacterial microcompartment organelle. Proc. Natl. Acad. Sci. USA 112, 2990-2995 (2015).

29. Crowley, C.S. et al. Structural insight into the mechanisms of transport across the Salmonella enterica Pdu microcompartment shell. J. Biol. Chem. 285, 37838-37846 (2010).

30. Pang, A., Frank, S., Brown, I., Warren, M.J. \& Pickersgill, R.W. Structural insights into higher order assembly and function of the bacterial microcompartment protein PduA. J. Biol. Chem. 289, 22377-22384 (2014).

31. Thomas, F., Boyle, A.L., Burton, A.J. \& Woolfson, D.N. A set of de novo designed parallel heterodimeric coiled coils with quantified dissociation constants in the micromolar to sub-nanomolar regime. J. Am. Chem. Soc. 135, 5161-5166 (2013).

32. Lee, M.J., Brown, I.R., Juodeikis, R., Frank, S. \& Warren, M.J. Employing bacterial microcompartment technology to engineer a shell-free enzymeaggregate for enhanced 1,2-propanediol production in Escherichia coli. Metab. Eng. 36, 48-56 (2016).

33. Fletcher, J.M. et al. Self-assembling cages from coiled-coil peptide modules. Science 340, 595-599 (2013).

34. Weber, B. et al. Automated tracing of microtubules in electron tomograms of plastic embedded samples of Caenorhabditis elegans embryos. J. Struct. Biol. 178, 129-138 (2012).

35. Johnson, E. et al. Correlative in-resin super-resolution and electron microscopy using standard fluorescent proteins. Sci. Rep. 5, 9583 (2015).

36. Mueller-Reichert, T. \& Verkade, P. Correlative Light and Electron Microscopy II; Methods in Cell Biology Vol. 124 (Academic Press, 2014).

37. Szeto, T.H., Rowland, S.L., Habrukowich, C.L. \& King, G.F. The MinD membrane targeting sequence is a transplantable lipid-binding helix. J. Biol. Chem. 278, 40050-40056 (2003).

38. Noël, C.R., Cai, F. \& Kerfeld, C.A. Purification and characterization of protein nanotubes assembled from a single bacterial microcompartment shell subunit. Adv. Mater. Interfaces 3, 1500295 (2015).

39. Lewicka, A.J. et al. Fusion of pyruvate decarboxylase and alcohol dehydrogenase increases ethanol production in Escherichia coli. ACS Synth. Biol. 3, 976-978 (2014). 


\section{Acknowledgments}

We are grateful to the Biotechnology and Biological Sciences Research Council of the UK for a strategic LoLa Award to M.J.W., D.N.W., P.V. and W.-F.X. (BB/M002969/1). D.N.W. holds a Royal Society Wolfson Research Merit Award. We thank the Wolfson Bioimaging Facility and BrisSynBio, a BBSRC/EPSRC-funded Synthetic Biology Research Centre (L01386X), for access to confocal and electron microscopes; K. Howland for assistance with GC-MS analysis; R. Sessions and I. Uddin for preparing images used in Supplementary Figure 1; L. Harrington and P. Schwille for advice on the MinD system; and the entire BMC-SAGE LoLa group for helpful discussions.

\section{Author contributions}

M.J.L. made constructs, prepared samples for TEM and confocal analysis, imaged samples by TEM, purified nanotubes and analyzed them by TEM and AFM and conducted the ethanol production experiments and analyses. J.M. undertook tomography and 3D reconstructions. L.H. undertook CLEM sample preparation and imaging. D.A. undertook confocal imaging. I.R.B. sectioned samples for TEM analysis. W.-F.X. assisted with AFM and statistical analysis. M.J.L., J.M., L.H., J.M.F., S.F., P.V., D.N.W. and M.J.W. designed the experiments. All authors contributed to the manuscript.

\section{Competing financial interests}

The authors declare no competing financial interests.

\section{Additional information}

Any supplementary information, chemical compound information and source data are available in the online version of the paper. Reprints and permissions information is available online at http://www.nature.com/reprints/index.html. Publisher's note: Springer Nature remains neutral with regard to jurisdictional claims in published maps and institutional affiliations. Correspondence and requests for materials should be addressed to M.J.W. or D.N.W. 


\section{ONLINE METHODS}

Cloning of coiled-coil fused constructs. DNA encoding CC-Di-A and CC-Di-B embedded within a GS linker followed by a hexahistidine tag and a thrombin cleavage sequence was synthesized and cloned into the XbaI/NdeI sites of pET14b. A control sequence containing only a GS linker, hexahistidine tag and thrombin cleavage sequence was also synthesized and cloned by the same strategy. Strains and synthesized DNA sequences with corresponding amino acid sequences are shown in Supplementary Tables $\mathbf{1}$ and 2. Plasmids and primers used in this study are outlined in Supplementary Tables 3 and 4.

Expression of coiled-coil constructs. E. coli BL21*(DE3)-competent cells were transformed with a plasmid(s) containing the gene(s) of interest and plated onto LB agar plates supplemented with appropriate antibiotics $(100 \mathrm{mg} / \mathrm{L}$ ampicillin and/or $34 \mathrm{mg} / \mathrm{L}$ chloramphenicol). For TEM analysis, $50 \mathrm{~mL}$ of LB was inoculated 1:100 from an overnight starter culture and grown at $37^{\circ} \mathrm{C}$ with shaking to an $\mathrm{OD}_{600}$ of $\sim 0.4$; protein production was induced by the addition of IPTG to a final concentration of $400 \mu \mathrm{M}$, and cultures were subsequently incubated overnight at $19^{\circ} \mathrm{C}$ with shaking. For time-course analysis, $500 \mathrm{~mL}$ of LB was inoculated, grown and induced as described above. At time intervals $50 \mathrm{~mL}$ of media was removed for TEM analysis. For purification of nanotubes, $250 \mathrm{~mL}$ LB was inoculated 1:100 from an overnight starter culture and grown at $37^{\circ} \mathrm{C}$ to an $\mathrm{OD}_{600} \sim 0.4$. Protein production was induced by addition of IPTG to a final concentration of $400 \mu \mathrm{M}$, and cultures were then incubated with shaking at $19^{\circ} \mathrm{C}$ overnight. For confocal imaging experiments $50 \mathrm{~mL}$ of LB was inoculated 1:100 from an overnight starter culture and grown with shaking at $37^{\circ} \mathrm{C}$ to an $\mathrm{OD}_{600} \sim 0.4$. protein production was induced by addition of IPTG to a final concentration of $400 \mu \mathrm{M}$, and cultures were then incubated with shaking at $19^{\circ} \mathrm{C}$ for $4 \mathrm{~h}$.

In vivo ethanol production. For in vivo ethanol production, $100 \mathrm{~mL}$ of LB supplemented with $4 \%$ glucose and appropriate antibiotics was inoculated from overnight starter cultures to a starting $\mathrm{OD}_{600}$ of 0.05 ; cultures were grown at $28^{\circ} \mathrm{C}$ for $120 \mathrm{~h}$ with shaking at $150 \mathrm{rpm}$. Protein production was induced by addition of IPTG to a final concentration of $400 \mu \mathrm{M}$ after $4 \mathrm{~h}$ of growth. During growth, $1 \mathrm{~mL}$ samples were removed at $0,2,4,6,12,24,48,72,96$ and $120 \mathrm{~h}$ for GC/MS analysis of the growth medium. Samples $(1 \mathrm{~mL})$ were also taken at each time point for SDS-PAGE analysis. Additional samples $(5 \mathrm{~mL})$ were taken after $24 \mathrm{~h}$ for TEM analysis.

Western blot analysis. Nitrocellulose membranes following transfer and blocking were incubated in primary antibody (rabbit anti-PduA, $1 \mu \mathrm{g} / \mathrm{mL}^{27}$, or mouse anti-His (Sigma-Aldrich; catalog \#H1029; diluted 1:3,000)), and then followed by incubation in a secondary antibody coupled to alkaline phosphatase (goat anti-rabbit IgG $(\mathrm{H}+\mathrm{L})$ alkaline phosphatase conjugate (Bio-Rad; catalog \#1706518; diluted 1:3,000) or anti-mouse IgG $(\mathrm{H}+\mathrm{L})$, AP conjugate (Promega; cata$\log \#$ S3721; diluted 1:5,000)). Bands were visualized by incubation in substrate 5-bromo-4-chloro-3-indolyl phosphate/nitro blue tetrazolium (BCIP/NBT).

Transmission electron microscopy (TEM) analysis of cells. Cells grown as described previously were harvested by centrifugation at 3,000 $\times g$ for $10 \mathrm{~min}$. The cell pellet was resuspended in $2 \mathrm{~mL} \mathrm{2.5 \% (w/v)} \mathrm{glutaraldehyde} \mathrm{in} 100 \mathrm{mM}$ sodium cacodylate buffer, $\mathrm{pH} 7.2(\mathrm{CAB})$, and fixed for $2 \mathrm{~h}$ with gentle rotating $(20 \mathrm{rpm})$. Cells were pelleted by centrifugation at $6,000 \times g$ for $2 \mathrm{~min}$ and were washed twice for $10 \mathrm{~min}$ with $100 \mathrm{mM} \mathrm{CAB}$. Cells were post-fixed with $1 \%(\mathrm{w} / \mathrm{v})$ osmium tetroxide in $100 \mathrm{mM} \mathrm{CAB}$ for $2 \mathrm{~h}$ and subsequently washed twice with $\mathrm{ddH}_{2} \mathrm{O}$. Cells were dehydrated by incubation in an ethanol gradient of $50 \% \mathrm{EtOH}$ for $10 \mathrm{~min}, 70 \% \mathrm{EtOH}$ overnight, and $90 \% \mathrm{EtOH}$ for 10 min followed by three $10 \mathrm{~min}$ washes in $100 \%$ dry EtOH. Cells were then washed twice with propylene oxide for $15 \mathrm{~min}$. Cell pellets were embedded by resuspension in $1 \mathrm{~mL}$ of a 1:1 mix of propylene oxide and Agar LV Resin and incubated for 30 min with rotation. Cell pellets were infiltrated twice in 100\% Agar LV resin. The cell pellet was resuspended in fresh resin and transferred to a $1 \mathrm{~mL}$ Beem embedding capsule, centrifuged for $5 \mathrm{~min}$ at $3,000 \times g$ to concentrate the cells to the tip of the mold and incubated for $20 \mathrm{~h}$ at $60{ }^{\circ} \mathrm{C}$ to polymerize.

Samples were ultra-thin sectioned on a RMC MT-XL ultra-microtome with a diamond knife $\left(\right.$ diatome $\left.45^{\circ}\right)$. Sections $(60-70 \mathrm{~nm})$ were collected on uncoated 300 mesh copper grids. Grids were stained by incubation in $4.5 \%(\mathrm{w} / \mathrm{v})$ uranyl acetate in $1 \%(\mathrm{v} / \mathrm{v})$ acetic acid solution for $45 \mathrm{~min}$ followed by washing in a stream of $\mathrm{ddH}_{2} \mathrm{O}$. Grids were then stained with Reynolds lead citrate for $7 \mathrm{~min}$, which was followed by washing in a stream of $\mathrm{ddH}_{2} \mathrm{O}$. Electron microscopy was performed using a JEOL-1230 transmission electron microscope equipped with a Gatan multiscan digital camera operated at an accelerating voltage of $80 \mathrm{kV}$

Tomography. Sections ( $250 \mathrm{~nm}$ ) were cut from the existing blocks, and $15 \mathrm{~nm}$ gold fiducials (Aurion, TomoSol solution) were applied to both surfaces of the sections. The sections were imaged at $200 \mathrm{kV}$ in a Tecnai 20 TEM (FEI, the Netherlands) and double tilt series images acquired between $-62^{\circ}$ to $+69.5^{\circ}$ (first axis) and $-68^{\circ}$ to $+69.5^{\circ}$ (second axis) with $1.5^{\circ}$ (above $50^{\circ}$ ) and $2^{\circ}$ increments (below $50^{\circ}$ ). The pixel size on the $4 \mathrm{k}$ by $4 \mathrm{k}$ FEI Eagle camera was $0.74 \mathrm{~nm}$. The resulting tomograms were reconstructed and combined using IMOD software ${ }^{40,41}$. The tube-like structures were modeled automatically using the AMIRA XTracing Extension of the AMIRA software suite, developed for automatic tracing of microtubules ${ }^{34}$. A cylinder template is correlated with the data to find and search for the center lines of tubes. A small cropped area was used to refine the fitting parameters as shown in Supplementary Video 2 and these were then applied to the full data set. AMIRA software was further used for visualizing the data.

Measurements of in vivo nanotubes. Diameter measurements of 100 nanotubes from 10 cells were calculated in Image ${ }^{42}$. Length measurements were calculated automatically using the XTracing extension of the AMIRA software suite. Cropping box measurements were removed manually from the data set, leaving a total of 739 tubes.

Purification of CC-Di-B-PduA* CC-Di-B-tagged PduA* was overproduced as described previously. Cells were harvested by centrifugation at $2,683 \times g$. A $1 \mathrm{~g}$ wet cell pellet was resuspended in $20 \mathrm{~mL}$ Yeast Protein Extraction Reagent (Thermo Scientific) supplemented with Protease Inhibitor Cocktail Tablets, EDTA-Free (Sigma-Aldrich) and 500 Units Benzonase Nuclease (Merck) and incubated for $3 \mathrm{~h}$ at room temperature with gentle shaking. CC-Di-B-PduA* nanotubes were pelleted from the lysate by centrifugation for $5 \mathrm{~min}$ at $11,300 \times \mathrm{g}$, and the pellet was resuspended in $2 \mathrm{~mL}$ of $20 \mathrm{mM}$ Tris- $\mathrm{HCl}, \mathrm{pH} 8$, containing $20 \mathrm{mM} \mathrm{NaCl}$. The suspension was centrifuged for $5 \mathrm{~min}$ at $11,000 \times g$, and the resulting nanotube containing pellet was resuspended in $20 \mathrm{mM}$ Tris- $\mathrm{HCl}$, $\mathrm{pH} 8$, and centrifuged again as above. The supernatant was removed and adjusted with a solution of $5 \mathrm{M} \mathrm{NaCl}$ to give a final concentration of $80 \mathrm{mM}$. A final centrifugation step as above was performed, and the resulting pellet was analyzed for the presence of $\mathrm{PduA}^{*}$ nanotubes.

Analysis of purified nanotubes. TEM: Following purification, $20 \mu \mathrm{L}$ of CCDi-B-PduA* nanotubes were deposited onto Formvar, carbon-coated 300 mesh copper grids and incubated to $5 \mathrm{~min}$. Glutaraldehyde $(20 \mu \mathrm{L}$ of $2.5 \%(\mathrm{v} / \mathrm{v}))$ in PBS was then added and incubated for a further $5 \mathrm{~min}$ before washing in three drops of $2.5 \%(\mathrm{v} / \mathrm{v})$ glutaraldehyde in PBS followed by three drops of $\mathrm{ddH}_{2} \mathrm{O}$. Grids were stained with $2 \%(\mathrm{w} / \mathrm{v})$ aqueous uranyl acetate and subsequently dried. Electron microscopy was performed using a JEOL-1230 transmission electron microscope equipped with a Gatan multiscan digital camera operated at an accelerating voltage of $80 \mathrm{kV}$.

AFM: Purified CC-Di-B-PduA ${ }^{\star}$ nanotubes $(20 \mu \mathrm{L})$ were deposited onto freshly cleaved mica surfaces and incubated for $5 \mathrm{~min}$, followed by the addition of $20 \mu \mathrm{L} 2.5 \%(\mathrm{v} / \mathrm{v})$ glutaraldehyde in PBS. Surfaces were washed three times with $1 \mathrm{~mL}$ of $\mathrm{ddH}_{2} \mathrm{O}$ then dried under a gentle stream of $\mathrm{N}_{2}$. Images were acquired in air at $20^{\circ} \mathrm{C}$ using a Bruker MultiMode 8 Scanning probe microscope operating under Peak-Force tapping mode (ScanAsyst, Bruker) with a ScanAsyst-air probe (Bruker). Areas $(10 \mu \mathrm{m} \times 10 \mu \mathrm{m})$ were scanned at a resolution of 4,096 $\times 4,096$ pixels. Bow and tilt were removed using NanoScope Analysis 1.4 (Bruker).

Confocal imaging. Following growth and induction of protein expression, $1 \mathrm{~mL}$ of cells was harvested by centrifugation at 3,000 $\times g$. The resulting cell pellet was washed three times in PBS before being incubated for $15 \mathrm{~min}$ in 
$2 \%(w / v)$ formaldehyde in PBS. Cells were then washed three more times in PBS. Cells $(10 \mu \mathrm{L})$ were pipetted onto a 1.5 thickness coverslip before being inverted onto a drop of ProLong Gold antifade mountant (Life Technologies) on a glass slide. Slides were incubated at room temperature in the dark for $24 \mathrm{~h}$ to cure. Images were acquired on a Leica TCS SP8 system attached to a Leica DMi8 inverted microscope (Leica Microsystems). Excitation light (514 nm for Citrine or $594 \mathrm{~nm}$ for mCherry) was provided by a white-light laser with a repetition rate of $80 \mathrm{MHz}$. Images were acquired using a $100 \times 1.4 \mathrm{NA}$ oil immersion objective and fluorescence was detected through bandpasses of 520-570 nm (Citrine detection) or 600-650 nm (mCherry detection).

Correlative light electron microscopy. Cells $(1 \mu \mathrm{L})$ were harvested by centrifugation at 3,000 $\times \mathrm{g}$ for $5 \mathrm{~min}$, and then loaded into a $0.1 \mathrm{~mm}$ membrane carrier (Leica) and vitrified by high pressure freezing (EMPACT2 + RTS, Leica). Frozen membrane carriers were transferred into $1 \mathrm{~mL}$ of freeze substitution medium $\left(0.2 \%\right.$ uranyl acetate, $5 \% \mathrm{H}_{2} \mathrm{O}$, in acetone) and held at $-90{ }^{\circ} \mathrm{C}$ for $5 \mathrm{~h}$ in an automated freeze substitution unit (AFS2, Leica) equipped with an attachment for automated reagent exchange (Freeze Substitution Processor, FSP, Leica). Samples were warmed to $-45^{\circ} \mathrm{C}$ at a rate of $5{ }^{\circ} \mathrm{C} / \mathrm{h}$, and held at $-45^{\circ} \mathrm{C}$ for $2 \mathrm{~h}$ before washing in acetone and ethanol for $30 \mathrm{~min}$ each. Samples were then infiltrated with $25 \%, 50 \%$ and $75 \%$ dilutions of Lowicryl HM20 resin for $3 \mathrm{~h}$ each before infiltrating with $100 \%$ resin overnight, which was followed by three further changes of resin for $2 \mathrm{~h}$ each. UV polymerization was performed over approximately $48 \mathrm{~h}$; Samples were initially kept at $-45^{\circ} \mathrm{C}$ for $16 \mathrm{~h}$, then warmed to $0{ }^{\circ} \mathrm{C}$ at a slope of $5^{\circ} \mathrm{C} / \mathrm{h}$, and finally kept at $0{ }^{\circ} \mathrm{C}$ for approximately $14 \mathrm{~h}$.

Following polymerization, blocks were removed from flow through containers, and carriers were detached using liquid nitrogen and the specimen carrier detaching tool (Leica) heated to $40^{\circ} \mathrm{C}$. Blocks were trimmed and sectioned with a $45^{\circ}$ diamond knife using an EM UC6 microtome (Leica). 70 - and 300-nm thick sections were collected on carbon-coated pioloform films on H6 copper finder grids (Agar Scientific). Grids were air dried, mounted in PBS between a glass slide and coverslip and imaged by light microscopy using a Leica DMI4000 B inverted epifluorescence microscope fitted with a $63 \times$ oil immersion lens (NA 1.4). After imaging, the grids were washed in $\mathrm{H}_{2} \mathrm{O}$ and air dried before imaging in TEM. Image registration of light and electron microscopy images was performed using the eC-CLEM plugin in $\mathrm{ICY}^{43}$.
Analysis of enzyme levels. Relative amounts of Pdc and Adh were quantified by western blot. Total cell lysate samples, adjusted to cell number were analyzed by SDS-PAGE and subsequently western blot analysis. Peak areas were quantified using the gel analysis tool in ImageJ. Due to the higher molecular weight band close to CC-Di-A-Adh, half of this peak was quantified with the assumption that the peak was symmetrical. Measurements were repeated for each of the cultures.

MinD colocalization. DNA encoding the c-terminal membrane-associating region of MinD was synthesized and cloned into the SpeI/BlpI sites of pET_ CC_Di_A_Citrine_No_Stop and pET_C_Citrine_No_Stop. Cells were transformed as described previously and grown in LB media at $37^{\circ} \mathrm{C}$ with shaking to an $\mathrm{OD}_{600} \sim 0.4$, protein production was induced by addition of IPTG to a final concentration of $400 \mu \mathrm{M}$. Cultures were then incubated with shaking at $19^{\circ} \mathrm{C}$ for $4 \mathrm{~h}$. Cells were harvested, fixed, embedded and sectioned as described previously. A total of 250 cells in the transverse orientation for each strain were analyzed for the presence and location of transverse CC-Di-B-PduA* nanotubes. Statistical analysis was performed in Minitab Software version 17 using a one-way ANOVA (analysis of variance) at the $99 \%$ level with post-hoc analysis by Tukey's test.

Life sciences reporting summary. Further information on experimental design and reagents is available in the Life Sciences Reporting Summary.

Data availability. All data generated or analyzed during this study are included in this published article (and supplementary information files) or are available from the corresponding authors on reasonable request.

40. Kremer, J.R., Mastronarde, D.N. \& McIntosh, J.R. Computer visualization of three-dimensional image data using IMOD. J. Struct. Biol. 116, 71-76 (1996).

41. Mastronarde, D.N. Dual-axis tomography: an approach with alignment methods that preserve resolution. J. Struct. Biol. 120, 343-352 (1997).

42. Schindelin, J. et al. Fiji: an open-source platform for biological-image analysis. Nat. Methods 9, 676-682 (2012).

43. Paul-Gilloteaux, P. et al. eC-CLEM: flexible multidimensional registration software for correlative microscopies. Nat. Methods 14, 102-103 (2017). 


\section{nature research}

Corresponding author(s): Dek Woolfson and Martin Warren

Initial submission $\searrow$ Revised version

Final submission

\section{Life Sciences Reporting Summary}

Nature Research wishes to improve the reproducibility of the work that we publish. This form is intended for publication with all accepted life science papers and provides structure for consistency and transparency in reporting. Every life science submission will use this form; some list items might not apply to an individual manuscript, but all fields must be completed for clarity.

For further information on the points included in this form, see Reporting Life Sciences Research. For further information on Nature Research policies, including our data availability policy, see Authors \& Referees and the Editorial Policy Checklist.

\section{- Experimental design}

1. Sample size

Describe how sample size was determined.

2. Data exclusions

Describe any data exclusions.

3. Replication

Describe whether the experimental findings were reliably reproduced.

\section{Randomization}

Describe how samples/organisms/participants were allocated into experimental groups.

5. Blinding

Describe whether the investigators were blinded to group allocation during data collection and/or analysis.

Note: all studies involving animals and/or human research participants must disclose whether blinding and randomization were used.

\section{Statistical parameters}

For all figures and tables that use statistical methods, confirm that the following items are present in relevant figure legends (or in the Methods section if additional space is needed).

n/a Confirmed

$\bigotimes$ The exact sample size $(n)$ for each experimental group/condition, given as a discrete number and unit of measurement (animals, litters, cultures, etc.)

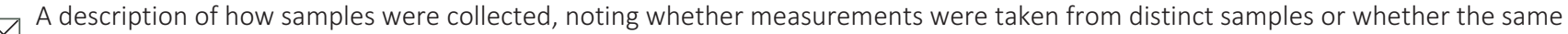
sample was measured repeatedly

$\bigotimes$ A statement indicating how many times each experiment was replicated

The statistical test(s) used and whether they are one- or two-sided (note: only common tests should be described solely by name; more complex techniques should be described in the Methods section)

A description of any assumptions or corrections, such as an adjustment for multiple comparisons

The test results (e.g. $P$ values) given as exact values whenever possible and with confidence intervals noted

A clear description of statistics including central tendency (e.g. median, mean) and variation (e.g. standard deviation, interquartile range) Clearly defined error bars

See the web collection on statistics for biologists for further resources and guidance. 
Policy information about availability of computer code

\section{Software}

Describe the software used to analyze the data in this study.

Statistical analysis was performed in Minitab Software version 17 using a one-way ANOVA (Analysis of Variance) at the $99 \%$ level with posthoc analysis by Tukey's test.

For manuscripts utilizing custom algorithms or software that are central to the paper but not yet described in the published literature, software must be made available to editors and reviewers upon request. We strongly encourage code deposition in a community repository (e.g. GitHub). Nature Methods guidance for providing algorithms and software for publication provides further information on this topic.

\section{- Materials and reagents}

Policy information about availability of materials

8. Materials availability

Indicate whether there are restrictions on availability of unique materials or if these materials are only available for distribution by a for-profit company.

\section{Antibodies}

Describe the antibodies used and how they were validated for use in the system under study (i.e. assay and species).

\section{Eukaryotic cell lines}

a. State the source of each eukaryotic cell line used.

b. Describe the method of cell line authentication used.

c. Report whether the cell lines were tested for mycoplasma contamination.

d. If any of the cell lines used are listed in the database of commonly misidentified cell lines maintained by ICLAC, provide a scientific rationale for their use.

All material will be made available upon request.

\section{NR \\ NR}

NR

$N R$

\section{- Animals and human research participants}

Policy information about studies involving animals; when reporting animal research, follow the ARRIVE guidelines

\section{Description of research animals}

Provide details on animals and/or animal-derived materials used in the study.

Policy information about studies involving human research participants

\section{Description of human research participants}

Describe the covariate-relevant population NR

characteristics of the human research participants. 